\title{
Multicenter, cross-sectional observational study of the impact of neuropathic pain on quality of life in cancer patients
}

\author{
So Yeon $\mathrm{Oh}^{1}$ • Sang Won Shin ${ }^{2} \cdot$ Su-Jin $\mathrm{Koh}^{3} \cdot$ Sang Byung Bae ${ }^{4} \cdot$ Hyun Chang ${ }^{5}$. \\ Jung Han Kim ${ }^{6}$ • Hyo Jung Kim ${ }^{7}$ • Young Seon Hong ${ }^{8}$ - Keon Uk Park ${ }^{9}$. \\ Jeanno Park ${ }^{10}$ - Kyung Hee Lee ${ }^{11}$ • Na Ri Lee ${ }^{12}$. Jung Lim Lee ${ }^{13}$ - Joung Soon Jang ${ }^{14}$. \\ Dae Sik Hong ${ }^{15}$. Seung-Sei Lee ${ }^{16}$. Sun Kyung Baek ${ }^{17}$ • Dae Ro Choi ${ }^{18}$. \\ Jooseop Chung ${ }^{19}$ - Sang Cheul $\mathrm{Oh}^{20}$ • Hye Sook Han ${ }^{21}$ • Hwan Jung Yun ${ }^{22}$. \\ Sun Jin $\mathrm{Sym}^{23}$ • So Young Yoon ${ }^{24}$ - In Sil Choi ${ }^{25}$ • Byoung Yong Shim ${ }^{26}$. \\ Seok Yun Kang ${ }^{27}$ • Sung Rok Kim ${ }^{28}$ • Hyun Joo Kim ${ }^{29}$
}

Received: 30 January 2017 / Accepted: 22 June 2017 / Published online: 8 July 2017

(C) The Author(s) 2017. This article is an open access publication

\begin{abstract}
Purpose Neuropathic cancer pain (NCP) is a common and potentially debilitating symptom in cancer patients. We investigated the prevalence of $\mathrm{NCP}$, as well as its management and association with QOL.
\end{abstract}

Presented in part at the MASCC/ISOO International Symposium on Supportive Care in Cancer, Miami, USA, June 26-28, 2014.

Electronic supplementary material The online version of this article (doi:10.1007/s00520-017-3806-5) contains supplementary material, which is available to authorized users.

Sang Won Shin

shinsw@kumc.or.kr

1 Internal Medicine, Pusan National University Yangsan Hospital, Yangsan, South Korea

2 Medical Oncology, Department of Internal Medicine, Korea University Anam Hospital, Korea University College of Medicine, 73, Inchon-ro, Seongbuk-gu, Seoul 136-705, Republic of Korea

3 Internal Medicine, Ulsan University College of Medicine, Ulsan, South Korea

4 Internal Medicine, Soonchunhyang University College of Medicine, Cheonan, South Korea

5 Internal Medicine, Seoul National University Bundang Hospital, Soengnam-Si, South Korea

6 Internal Medicine, Kangnam Sacred Heart Hospital, Hallym University Medical Center, Hallym University College of Medicine, Seoul, South Korea

7 Internal Medicine, Hallym University Sacred Heart Hospital, Anyang, South Korea
Methods Cancer patients with pain $\geq 1$ on the visual analogue scale (VAS) were surveyed with the Douleur Neuropathique (DN4) questionnaire, the Brief Pain Inventory-Short Form (BPI-SF), and the EuroQOL five dimensions (EQ-5D) questionnaire. The associations between $\mathrm{NCP}$ and pain severity

8 Internal Medicine, Seoul St. Mary's Hospital, The Catholic University of Korea, Seoul, South Korea

9 Internal Medicine, Dongsan Medical Center, Keimyung University, Daegu, South Korea

10 Internal Medicine, Bobath Memorial Hospital, KyungGi, Soengnam-Si, South Korea

11 Internal Medicine, Yeungnam University College of Medicine, Daegu, South Korea

12 Internal Medicine, Chonbuk National University College of Medicine, Chonbuk, South Korea

13 Internal Medicine, Daegu Fatima Hospital, Daegu, South Korea

14 Internal Medicine, Chung-Ang University College of Medicine, Seoul, South Korea

15 Internal Medicine, Soonchunhyang University Bucheon Hospital, Bucheon, South Korea

16 Internal Medicine, Sungkyunkwan University College of Medicine Kangbuk Samsung Hospital, Seoul, South Korea

17 Internal Medicine, Kyung Hee University Medical Center, Seoul, South Korea 
or NCP and QOL, while controlling for variables relevant to QOL, were then analyzed.

Results A total of 2003 patients were enrolled in this survey; the prevalence of NCP was $36.0 \%(n=722,95 \%$ CI, 32.539.5 ). We found that NCP in cancer patients was closely correlated to a higher pain severity (BPI-SF; $4.96 \pm 1.94$ versus $4.24 \pm 2.02, p<0.001$ ), and in patients with $\mathrm{NCP}$, pain more severely interfered with daily living, as compared to those without NCP (BPI-SF; $4.86 \pm 2.71$ versus $4.41 \pm 2.87$, $p<0.001$ ). Patients with NCP also had worse QOL than those without NCP, as measured by EQ-5D index score $(0.47 \pm 0.30$ vs. $0.51 \pm 0.30, p=0.005)$, and this was confirmed using multivariate analysis $(p<0.001)$, even after controlling for other variables such as age, sex, disease stage, cancer duration, radiotherapy, chemotherapy, and comorbidities. Importantly, adjuvant analgesics were used in less than half of patients with $\operatorname{NCP}(n=358,46.4 \%)$.

Conclusions We found that NCP in cancer patients was significantly associated with a worsened QOL, and current management is inadequate. Therefore, future research aimed at developing improved strategies for management of NCP is required.

Keywords Neuropathic pain · Quality of life · Neoplasm • Pain management

\footnotetext{
18 Internal Medicine, Hallym University Chuncheon Medical Center, Chuncheon, South Korea

19 Internal Medicine, Pusan National University Hospital, Pusan, South Korea

20 Internal Medicine, Korea University Guro Hospital, Seoul, South Korea

21 Internal Medicine, Chungbuk National University College of Medicine, Chungbuk, South Korea

22 Internal Medicine, Chungnam National University College of Medicine, Chungnam, South Korea

23 Internal Medicine, Gachon University Gil Medical Center, Incheon, South Korea

24 Internal Medicine, Konkuk University Medical Center, Seoul, South Korea

25 Internal Medicine, SMG-SNU Boramae Medical Center, Seoul, South Korea

26 Internal Medicine, St. Vincent's Hospital, The Catholic University of Korea, Suwon, South Korea

27 Department of Hematology-Oncology, Ajou University School of Medicine, Suwon, South Korea

28 Internal Medicine, Inje University Sanggye Paik Hospital, Seoul, South Korea

29 Corporate Affairs \& Health and Value, Pfizer Pharmaceutical Korea Ltd., Seoul, South Korea
}

\section{Introduction}

Pain has been recognized as a key symptom in various types of cancer, and it is the most common symptom leading to a cancer diagnosis (in about $30 \%$ of patients) [1]. Although recent guidelines have emphasized the treatment of pain in cancer patients, it remains undertreated $[2,3]$. It has also been found that cancer patients with pain have significantly lower levels of performance status and higher levels of anger, fatigue, depression, confusion, and lethargy, as compared to those cancer patients who did not experience pain, even after accounting for disease stage [4].

Neuropathic pain may adversely affect quality of life (QOL) in cancer patients and could also increase care difficulty $[5,6]$. Previous studies have investigated the impact of NCP on QOL [6-9]. But these mostly focused on chemotherapyinduced peripheral neuropathy (CIPN) alone in specific disease conditions. NCP is particularly important to diagnose because distinct treatment strategies are required that differ from those needed for nociceptive pain [10-12]. Critically, the features associated with NCP, including, but not limited to CIPN, especially among cancer patients, are poorly understood. To obtain a comprehensive insightful overview of NCP in cancer patients, it will be necessary to investigate the characteristics of a large group of general cancer patients with NCP, including those in all stages of disease, regardless of the treatments they have received.

This study was performed on behalf of Korean Cancer Study Group Neuropathic Cancer Pain Survey and was designed to assess the current status of NCP in cancer patients. We aimed to accomplish three main objectives: (i) determine the prevalence and associated characteristics of NCP in patients with cancer pain, (ii) identify the current patterns of management for NCP, and (iii) assess the association of NCP with QOL. We found that NCP was associated with a lower QOL in cancer patients, even after controlling for other potentially confounding variables, and it also remains undertreated, highlighting the need for improved pain management strategies.

\section{Methods}

\section{Study design}

This observational study is non-interventional, multicenter, and cross-sectional. The study was performed between February 2013 and March 2014 in the oncology clinic of 28 general hospitals, representing the general cancer patient population in the Republic of Korea. The first step of the study procedure was to interview and screen individual patients by medical personnel. Informed consent was then obtained from all eligible patients. Patients who signed the consent were next 
asked to complete a survey questionnaire about pain and QOL. Physicians evaluated the patients to determine the presence of neuropathic pain, and both demographic and clinical information was retrieved from the medical records. Lastly, the questionnaires and case report forms were collected, and the study data were analyzed. Central institutional review board (IRB) of Korean Cancer Study Group (KCSG) approved this study (study ID: KCSG PC13-02). After central approval, institutional approval was obtained again by every institution of the participating investigators.

\section{Patients}

A total of 2003 patients were enrolled in the survey. Inclusion criteria were patients who (1) were aged 20 years or older, (2) were diagnosed with cancer, (3) had pain with a visual analogue scale (VAS) measurement of one or higher, and (4) could understand and sign the informed consent. Patients were excluded if they had pain that is unrelated to cancer as per a physician's discretion.

\section{Data collection and measurement}

Clinical information potentially related to pain or neuropathic pain, including past medical histories, were collected from patient medical records. Current pain control status was evaluated by reviewing all drugs administered during the preceding 6 weeks. The presence of neuropathic pain was determined using the DN4 questionnaire where a total score of 4 or higher was defined as NCP in this study. The questionnaire consists of 10 items, 7 items related to pain quality which are based on an interview with the patient, and 3 items which are based on the clinical examination. A score of 1 is given to each positive item and a score of 0 to each negative item. The total score is calculated as the sum of all 10 items, and the cutoff value for the diagnosis of neuropathic pain is a total score of 4 out of 10 [13]. Pain characteristics were further evaluated using the Brief Pain Inventory-Short Form (BPI-SF) [14], Korean version [15]. BPI-SF is a self-administered questionnaire used to evaluate pain on its severity and its impact on the patient's daily functioning. Items in the pain severity evaluate the pain "at its worst," "at its least," and "on average" over the previous $24 \mathrm{~h}$, as well as pain at the time of completing the questionnaire. On the other hand, the pain interference scale asks the patient to rate how their pain interferes with their enjoyment of life, general activity, walking ability, mood, sleep, normal work, and relationships with other people. Patients respond on a 0 to 10 numerical scale where higher scores indicate higher level of pain and interference [14, 15]. Health-related QOL was measured using the Korean version of the EuroQOL five dimensions (EQ-5D) and the EQ-5D visual analogue scale (VAS) [16-18]. The EQ-5D index score consisted of five metrics, including mobility, self-care, usual activities, pain/discomfort, and anxiety/depression, with three grades of severity for each item. The EQ-5D index score ranges from 0 to 1 , with larger values indicating a better QOL. The EQ-5D VAS is simple thermometer-like bar scale, in which a patient can draw a line between 0 (worst) and 100 (best) indicating his or her overall condition, which is a gross self-assessment of general health status of the day that the survey is administered.

\section{Statistical analyses}

We estimated a target sample size based on the assumption that the prevalence of neuropathic pain is $30 \%$ [19]. With a significance level of 0.05 and an estimated error rate of $2 \%$, the required number of patients to be enrolled was calculated to be about 2000:

$$
\begin{aligned}
n & =p(1-p)\left(\frac{z a / 2}{d}\right)^{2}, p=0.3, Z a / 2=1.96 \text { when } a \\
& =0.05, \quad d=0.02 .
\end{aligned}
$$

For the data analysis, descriptive statistics were performed to determine the demographic and clinical characteristics of patients (Table 1). A Student's $t$ test was performed to compare the mean differences in pain level and QOL between the NCP and non-NCP groups (Tables 2 and 4). Univariate regression analyses were performed to explore the factors associated with QOL (EQ5D) (Table 3). Using the determinants that were significantly ( $p$ value $<0.05$ ) associated with EQ5D in this univariate analysis, we performed multiple regression analysis. To determine if there were any interactions between two independent variables, we performed multiple regression analysis including sex ( $p$ value 0.338 ), other statistically significant $(p$ value $<0.05$ ) variables, and potential interaction terms. Among all multiple regression models, we selected the model with the smallest Akaike information criterion value. All statistical analyses were performed using IBM SPSS Statistics 20.0 (IBM Corporation, NY, USA).

\section{Results}

\section{Patient characteristics}

Demographic and clinical characteristics of all patients are presented in Table 1. The majority of patients had advancedstage cancer $(71.3 \%)$ and had or were receiving chemotherapy $(87.5 \%)$. Among comorbidities, the prevalence of diabetes (15.4\%) appeared to be the highest among our study population. Other comorbidities relevant to neuropathic pain were rare. For management of pain, opioid analgesics were most 
Table 1 Characteristics of study participants

\begin{tabular}{|c|c|}
\hline Patient data & $\begin{array}{l}\text { Total } n=2003 \\
n(\%)\end{array}$ \\
\hline \multicolumn{2}{|l|}{ Gender } \\
\hline Male & 1089 (54.4) \\
\hline Female & $914(45.6)$ \\
\hline Age, median (range) & $61.0(21-94)$ \\
\hline Time from diagnosis of cancer (months), median (range) & $13.0(1-336)$ \\
\hline \multicolumn{2}{|l|}{ Stage } \\
\hline 1 & $46(2.3)$ \\
\hline 2 & $103(5.1)$ \\
\hline 3 & $250(12.5)$ \\
\hline 4 & $1428(71.3)$ \\
\hline Unknown & $176(8.8)$ \\
\hline \multicolumn{2}{|l|}{ Comorbidities and history ${ }^{\mathrm{a}, \mathrm{b}}$} \\
\hline Diabetes & $309(15.4)$ \\
\hline Liver cirrhosis & $44(2.2)$ \\
\hline Traumatic injury & $43(2.1)$ \\
\hline Herpes zoster & $41(2.0)$ \\
\hline \multicolumn{2}{|l|}{ Management of pain ${ }^{\mathrm{a}}$} \\
\hline Opioid analgesics & $1313(65.6)$ \\
\hline Non-opioid analgesics & $748(37.3)$ \\
\hline Anticonvulsants & $464(23.2)$ \\
\hline Antidepressants & $134(6.7)$ \\
\hline Corticosteroids & $91(4.5)$ \\
\hline Benzodiazepines & $68(3.4)$ \\
\hline Others & $48(2.4)$ \\
\hline None as analgesic & $257(12.8)$ \\
\hline \multicolumn{2}{|l|}{ Experienced treatment ${ }^{\mathrm{a}}$} \\
\hline Chemotherapy & $1753(87.5)$ \\
\hline Surgery & $799(39.9)$ \\
\hline Radiation & $602(30.1)$ \\
\hline None & $220(11.0)$ \\
\hline \multicolumn{2}{|l|}{ Chemotherapeutic agents ${ }^{\mathrm{a}, \mathrm{c}}$} \\
\hline Alkylating agents & $1209(60.4)$ \\
\hline Taxanes & $485(24.2)$ \\
\hline Vinca alkaloids & $150(7.5)$ \\
\hline Others & $1500(74.9)$ \\
\hline \multicolumn{2}{|l|}{ Primary and metastatic sites of cancer ${ }^{\mathrm{a}}$} \\
\hline Gastrointestinal tract & $982(49.0)$ \\
\hline Respiratory system & $490(24.5)$ \\
\hline Skins, bones, connective tissue & $288(14.4)$ \\
\hline Lymphatic-hematopoietic system & $272(13.6)$ \\
\hline Genitourinary system & $269(13.4)$ \\
\hline Breast & $221(11.0)$ \\
\hline Head and neck region & $188(9.4)$ \\
\hline Others/multiple primary & $70(3.5)$ \\
\hline Unknown & $6(0.3)$ \\
\hline
\end{tabular}

${ }^{\text {a }}$ Permitted overlap

${ }^{\mathrm{b}}$ Comorbidities with incidence of less than $2 \%$ were not presented in this table

${ }^{\mathrm{c}} N=1751$ (missing $N=2$, those who did not select type of chemotherapeutic agents were excluded). Others include antimetabolites, topoisomerase inhibitors, cytotoxic antibiotics, etc. commonly used among all patients $(65.6 \%)$, followed by nonopioid analgesics $(37.3 \%)$ and anticonvulsants $(23.2 \%)$.

\section{Prevalence and management of neuropathic pain}

NCP was present in 722 out of the 2003 patients surveyed, and thus the prevalence of NCP was found to be $36.0 \%$ (95\% CI, 32.5-39.5). Among the patients diagnosed with NCP $(N=722)$, in most cases, it was associated with chemotherapy;
668 patients $(92.5 \%)$ had received or were receiving chemotherapy, while 54 patients $(7.5 \%)$ had never received chemotherapy. The prevalence of NCP was significantly higher among patients who had received or were receiving chemotherapy ( $n=1753$ ) compared to its prevalence of those who have never received chemotherapy $(n=250)$ (38.1 versus $21.6 \%, p<0.001$, Supplementary Appendix Table 1). Patients with moderate to severe pain (VAS $\geq 4, n=497$ ) had a higher prevalence of NCP than those with mild pain 
( $n=225$ ) (42.4 vs. $27.1 \%$ ) (Fig. 1). However, fewer than half of the patients with both moderate to severe pain and NCP were being treated with adjuvant analgesics targeting the NCP ( $n=358,49.6 \%$ ) (Fig. 1), although these were more frequently prescribed in this group than to those without NCP $(23.0 \%)$ $\left(p<0.001\right.$ by $\chi^{2}$ test, details in Supplementary Appendix Fig. 1).

\section{Prevalence and management of cancer pain}

More than half of the patients ( $n=1173,58.6 \%)$ had moderate to severe pain (VAS $\geq 4$ ), implying that cancer pain was not being adequately managed. Contrary to NCP, the portion of severe pain was significantly higher among patients who have never received chemotherapy $(n=250)$ compared to those had received or were receiving chemotherapy $(n=1753)$ ( $p=0.010$, Supplementary Appendix Table 1). Pharmacologic management for the pain was presented at Table 1. Even though all patients participating in this study were those who reported some degree of cancer pain, still $12.8 \%(n=257)$ of the total patients were not prescribed any analgesic. When comparing pain treatment patterns in patients with or without NCP, we found that anticonvulsants, antidepressants, and benzodiazepines were more commonly used in patients with NCP then patients without NCP, whereas opioids, non-opioids, and corticosteroids were more commonly used in patients without NCP.

\section{NCP association with pain severity and QOL}

The pain severity score from the BPI-SF was measured in all patients; however, the pain interference score could not be measured in two patients. All patients answered the EQ-5D VAS, but six did not complete the EQ-5D index score questionnaire. Table 2 shows the differences in the mean pain and QOL scores in those with or without NCP. Patients with NCP had higher pain severity scores $(p<0.001)$ and higher pain interference scores $(p<0.001)$ in their daily living, than those without NCP. The QOL, as measured by the EQ-5D index score, was significantly worse in patients with NCP, than in those without NCP $(p=0.005)$. When analyzed by subscales of the EQ-5D, patients with NCP had a greater number of extreme problems than those without NCP in both the pain/ discomfort and the anxiety/depression domain $(p<0.001$ and $p=0.007$, respectively). Proportion distributions by each level of mobility, self-care, and usual activities domains were not significantly different between patients with and without NCP (Supplementary Appendix Table 2).

\section{Factors associated with QOL}

We analyzed demographic and clinical factors potentially affecting the EQ-5D index score and found that younger age $(p<0.001)$, shorter duration of cancer $(p=0.015)$, absence of comorbidities $(p<0.001)$, and absence of neuropathic pain $(p=0.005)$ were associated with better QOL scores in our univariate analysis. Conversely, patients with stage IV cancers $(p<0.001)$, those who never received chemotherapy $(p<0.001)$, or those who underwent radiotherapy $(p<0.001)$ had worse QOL status (Table 3). Even after a multivariate analysis, a diagnosis of NCP was among the factors that significantly affected QOL $(p<0.001)$; EQ-5D index score was 0.442 in patients with NCP and 0.497 in patients without NCP $(p<0.001)$, after adjusting for other variables.

\section{The effect of adjuvant analgesics targeting NCP on pain and QOL}

We investigated the mean pain and QOL score differences in patients receiving adjuvant analgesics targeting NCP and in those who did not. We found that patients with NCP who were managed with adjuvant analgesics had less interference with their daily living $(p=0.041)$ and had a better QOL (by EQ-5D VAS score) $(p=0.043)$ (Table 4$)$. If we again examine the details of the EQ-5D index score, a smaller proportion of patients having extreme problems were observed in the group receiving adjuvant analgesics, as compared to the group that were not receiving adjuvant analgesics in the mobility, selfcare, and usual activities domains $(p=0.028,0.031$, and 0.005 , respectively, linear-by-linear association). Proportion distributions in the pain/discomfort and anxiety/depression
Table 2 Pain and QOL scores in patients with and without $\mathrm{NCP}$

\begin{tabular}{lllll}
\hline Mean \pm SD & Total $(n=2003)$ & NCP $(n=722)$ & Non-NCP $(n=1281)$ & $p^{\mathrm{a}}$ \\
\hline Pain VAS at screening & $4.37 \pm 2.27$ & $4.90 \pm 2.27$ & $4.08 \pm 2.01$ & $<0.001$ \\
Pain Severity from BPI-SF & $4.50 \pm 2.02$ & $4.96 \pm 1.94$ & $4.24 \pm 2.02$ & $<0.001$ \\
Pain Interference score from BPI-SF & $4.57 \pm 2.82$ & $4.86 \pm 2.71$ & $4.41 \pm 2.87$ & $<0.001$ \\
EQ-5D index score & $0.49 \pm 0.30$ & $0.47 \pm 0.30$ & $0.51 \pm 0.30$ & 0.005 \\
EQ-5D VAS & $57.42 \pm 30.48$ & $56.53 \pm 21.42$ & $57.92 \pm 34.55$ & 0.327 \\
\hline
\end{tabular}

$S D$ standard deviation, $N C P$ neuropathic cancer pain, $V A S$ visual analogue scale

${ }^{\text {a }} p$ value by Student's $t$ test 
Table 3 Univariate and multivariate analyses of factors associated with QOL (EQ-5D index score)

\begin{tabular}{|c|c|c|c|c|}
\hline & \multicolumn{2}{|c|}{ Univariate analysis } & \multicolumn{2}{|c|}{ Multivariate analysis } \\
\hline & $B^{\mathrm{a}}$ & $p^{\mathrm{a}}$ & $B^{\mathrm{b}}$ & $p^{\mathrm{b}}$ \\
\hline Sex, male $(\text { female })^{\mathrm{c}}$ & 0.013 & 0.338 & 0.026 & 0.067 \\
\hline Age, $\geq 60(<60)$ & -0.065 & $<0.001$ & -0.055 & 0.000 \\
\hline Duration of cancer, $\geq 13$ months ( $<13$ months) & -0.033 & 0.015 & -0.024 & 0.102 \\
\hline Stage, IV (stages I-III) & -0.110 & $<0.001$ & -0.102 & 0.000 \\
\hline Chemotherapy, done (never done) & 0.116 & $<0.001$ & 0.069 & 0.000 \\
\hline Radiotherapy, done (never done) & -0.048 & 0.001 & -0.050 & 0.001 \\
\hline Surgery, done (never done) & 0.020 & 0.137 & & \\
\hline Comorbidities, present (none) & -0.085 & $<0.001$ & -0.072 & 0.000 \\
\hline Diagnosis of NCP, DN4 $\geq 4(\mathrm{DN} 4<4)$ & -0.039 & 0.005 & -0.053 & 0.000 \\
\hline NCP-targeted therapy, present (none) & -0.012 & 0.421 & & \\
\hline
\end{tabular}

${ }^{\text {a }}$ By regression analysis

${ }^{\mathrm{b}}$ By multiple regression analysis, $R^{2}=0.065$

${ }^{\mathrm{c}}$ Reference variables are in parenthesis domains were not significantly different between the two groups (Supplementary Appendix Table 3).

\section{Discussion}

In this study, we clearly found that patients with NCP have a worse QOL than those without NCP. This difference is statistically significant, even after controlling for other variables affecting QOL. In addition to NCP, older age, advanced cancer stage, never having received chemotherapy, have received radiotherapy, and presence of comorbidities were associated with low QOL scores. This is particularly meaningful, as we included patients who never received chemotherapy in our survey.
To the best of our knowledge, this is the first study that clearly demonstrates the relationship between NCP and QOL in a population of cancer patients encompassing a broad array of conditions. Apart from studies on CIPN, those that directly assessed QOL differences in various cancer patients with or without NCP are rare. As we expected, we observed that regardless of receiving chemotherapy or not, diagnosis of NCP was a significant factor that lowered patients QOL. Our study population consisted mainly of incurable advancedstage cancer patients, as well as patients undergoing treatment and cancer survivors. By doing this, we tried to obtain a global perspective of the general features of neuropathic pain in cancer patients and clarify its association with QOL.

One recently published study showed that neuropathic symptoms, such as numbness and tingling, are associated with poor QOL [6]. However, this study focused on CIPN in
Fig. 1 Prevalence of NCP according to pain intensity. NCP was more prevalent in patients with moderate to severe pain than in those with mild cancer pain (comparison between doublelined boxes; $p<0.001$ by $\chi^{2}$ test). Among patients having pain with a severity of VAS $\geq 4$, less than half were treated with adjuvant analgesics targeting NCP (lower boxes). Treatment with adjuvant analgesics targeting NCP was defined as the administration of antidepressants, anticonvulsants, corticosteroids, benzodiazepines, and with or without opioid or non-opioid analgesics

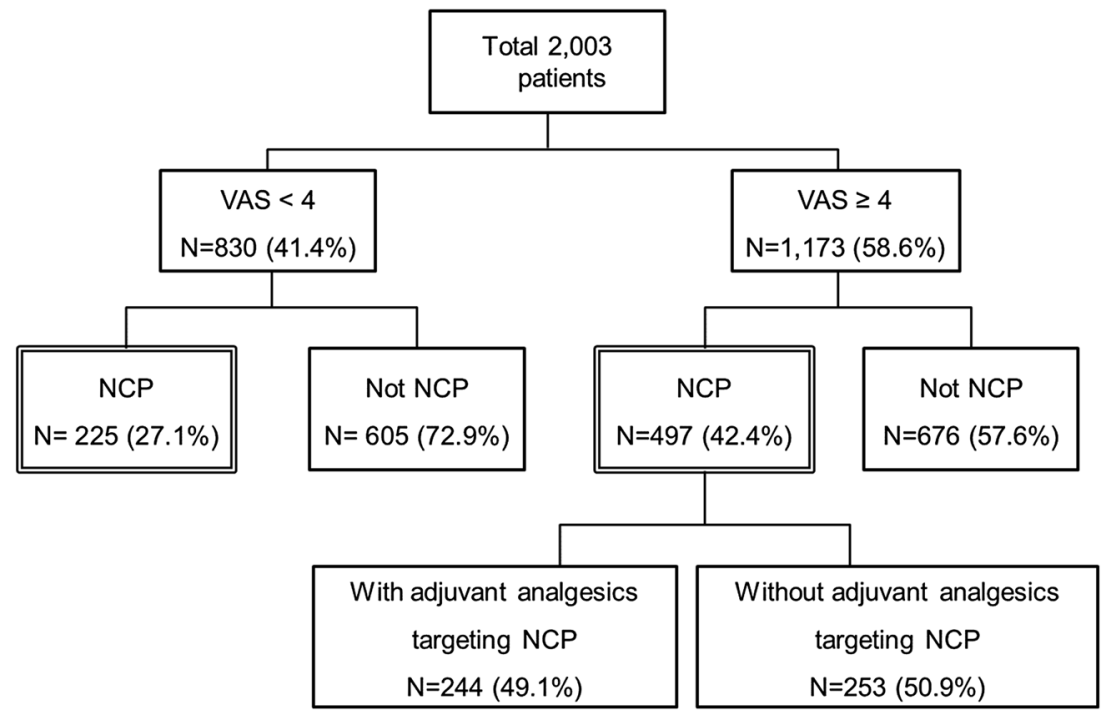


Table 4 Comparison of pain and QOL scales in patients diagnosed with NCP, with and without adjuvant analgesics targeting NCP

\begin{tabular}{llll}
\hline Mean \pm SD & \multicolumn{2}{l}{ Total $(n=772)$} & $p^{\text {a }}$ \\
\cline { 2 - 3 } & $\begin{array}{l}\text { With adjuvant analgesics targeting } \\
\text { NCP }(n=358)\end{array}$ & $\begin{array}{l}\text { Without adjuvant analgesics } \\
\text { targeting NCP }(n=364)\end{array}$ & 0.372 \\
\hline Pain VAS at screening & $4.82 \pm 2.25$ & $4.98 \pm 2.29$ & 0.262 \\
$\begin{array}{l}\text { Pain Severity from } \\
\quad \text { BPI-SF }\end{array}$ & $4.88 \pm 1.95$ & $5.04 \pm 1.93$ & 0.041 \\
$\begin{array}{l}\text { Pain Interference score } \\
\text { from BPI-SF }\end{array}$ & $4.66 \pm 2.63$ & $5.07 \pm 2.78$ & 0.090 \\
$\begin{array}{l}\text { EQ-5D index score } \\
\text { EQ-5D VAS }\end{array}$ & $0.49 \pm 0.28$ & $0.45 \pm 0.31$ & 0.043 \\
\hline SD standard deviation, $N C P$ neuropathic cancer pain & $54.93 \pm 22.84$ & \\
${ }^{a} p$ value by Student's $t$ test & $58.15 \pm 19.77$ & &
\end{tabular}

patients receiving chemotherapy. There are many additional studies that have examined the relationship between CIPN and QOL, and the negative association between the two is wellestablished. According to a recent systematic review [20], out of 11 studies that assessed the relationship between CIPN and QOL, eight showed a correlation between CIPN and worsened QOL [8, 21-26]. The remaining three studies did not find an association between CIPN and QOL. Among those eight studies that reported an association between CIPN and a lower QOL, one recent large-scale study showed that out of 1643 colorectal cancer survivors, those who reported to have a greater number of neuropathy symptoms (upper 10\%) had a statistically significantly worse QOL, as compared to those with fewer neuropathy symptoms (lower 90\%) [8]. Other studies demonstrated the association of CIPN and worse QOL in patients with colorectal cancer [7], lung cancer [21], lymphoma [24], ovarian cancer [26], and various solid tumors [23].

In this study, neuropathic cancer pain was diagnosed in 722 patients, out of 2003 patients surveyed, and the estimated prevalence was $36 \%$. This was similar to result from recent systematic review reporting that the prevalence of pain with neuropathic component was $39.1 \%$ (95\% CI, 28.9\%-49.5\%) [27]. More recently, a European survey estimated an occurrence rate of $32.6 \%$ (95\% CI, 29.62-35.58) for cancer-related neuropathic pain among cancer patients having chronic pain [28].

The etiology of NCP has not been fully defined. Although according to a systematic review [27], only $20.3 \%$ of cases were attributed to cancer treatment, a recently published study suggests that a larger portion of neuropathic pain originates from anticancer treatment (68.9\% tumor-related, $42.9 \%$ treatment-related) [29]. In our present study, most patients with NCP had received, or had been receiving chemotherapy $(87.5 \%)$, implying that a larger portion of NCP may be caused by anticancer treatments than previously known.

Although it is recommended that anticonvulsants or antidepressants are used in combination with opioid analgesics for management of NCP [30-32], we found that adjuvant analgesics, such as anticonvulsants, antidepressants, corticosteroids, benzodiazepines, or phenothiazines, were rarely used for the purpose of pain control in our study population. Less than half of patients with NCP were prescribed adjuvant analgesics, along with opioids or non-opioid analgesics. This proportion is not increased, even in patients experiencing moderate-to-severe pain and NCP, and this suggests that physicians should pay increased attention to pain in their cancer patients.

Our study has several limitations. First, in spite of the clear association between NCP and worsened QOL, we could not confirm a causal relationship between NCP and QOL, due to our cross-sectional study design. Additionally, although there was a statistical significance between NCP and non-NCP patients, the QOL difference was relatively small. Second, the population included in this study was heterogeneous; we enrolled patients who were receiving anticancer treatments, those who were under palliative care after finishing chemotherapy, and those who never underwent chemotherapy. This is what distinguishes our study from the other studies, which mostly focuses on CIPN in patients receiving a specific chemotherapy regimen. Such heterogeneity may attenuate or potentiate the association between NCP and QOL. By virtue of this heterogeneity however, this study can provide a general overview of the neuropathic pain in the cancer patients until more evidence on NCP etiology is available. Third, due to our cross-sectional study design, patients having a DN4 score $<4$ included both of patients who had never had NCP and those who may have experienced NCP but its symptoms were improved after management. From our study results, it is impossible to distinguish the two. Fourth, cutoff for poorly controlled pain was NRS score of 4, which was arbitrarily decided by the investigators instead of reflecting patients' personalized pain goal. And because we included patients who were not receiving any analgesics, the population without analgesics may have affected the overall outcome, such as the relationship between NCP and QOL. 
In summary, this study provides a clinically meaningful overview of neuropathic pain in patients with cancer pain. We found that there was a clear association between NCP and both increased pain severity and worsened QOL. These results suggest the need for prospective study aimed at clarifying the causality between neuropathic pain and worsened QOL. Furthermore, in general, pain was not adequately managed in our study population, and NCP-targeting drugs are not widely used. Therefore, new management strategies with improved efficacy are required, as current pharmacological management is not sufficient to alleviate neuropathic pain, and efforts to elucidate the detrimental effect of neuropathic pain will be meaningless, in the absence of effective methods to improve it.

Acknowledgments We appreciate SookJung Hyun, statistician at Primecore Consulting, for her statistical support.

\section{Compliance with ethical standards}

Conflict of interest The authors have no conflicts of interest to declare.

Funding This research was sponsored by Pfizer.

Open Access This article is distributed under the terms of the Creative Commons Attribution-NonCommercial 4.0 International License (http:// creativecommons.org/licenses/by-nc/4.0/), which permits any noncommercial use, distribution, and reproduction in any medium, provided you give appropriate credit to the original author(s) and the source, provide a link to the Creative Commons license, and indicate if changes were made.

\section{References}

1. Breivik H, Cherny N, Collett B, et al. (2009) Cancer-related pain: a pan-European survey of prevalence, treatment, and patient attitudes. Ann Oncol : mdp001

2. Deandrea S, Montanari M, Moja L, Apolone G (2008) Prevalence of undertreatment in cancer pain. A review of published literature. Ann Oncol 19:1985-1991

3. Hong SH, Roh SY, Kim SY et al (2011) Change in cancer pain management in Korea between 2001 and 2006: results of two nationwide surveys. J Pain Symptom Manag 41:93-103

4. Lin C-C, Lai Y-L, Ward SE (2003) Effect of cancer pain on performance status, mood states, and level of hope among Taiwanese cancer patients. J Pain Symptom Manag 25:29-37

5. Hershman D, Weimer L, Wang A et al (2011) Association between patient reported outcomes and quantitative sensory tests for measuring long-term neurotoxicity in breast cancer survivors treated with adjuvant paclitaxel chemotherapy. Breast Cancer Res Treat 125:767-774

6. Jones D, Zhao F, Brell J et al (2014) Neuropathic symptoms, quality of life, and clinician perception of patient care in medical oncology outpatients with colorectal, breast, lung, and prostate cancer. J Cancer Surviv:1-10

7. Padman S, Lee J, Kumar R et al (2015) Late effects of oxaliplatininduced peripheral neuropathy (LEON) - cross-sectional cohort study of patients with colorectal cancer surviving at least 2 years. Support Care Cancer 23:861-869
8. Mols F, Beijers T, Lemmens V, van den Hurk CJ, Vreugdenhil G, van de Poll-Franse LV (2013) Chemotherapy-induced neuropathy and its association with quality of life among 2- to 11-year colorectal cancer survivors: results from the population-based PROFILES registry. J Clin Oncol 31:2699-2707

9. Ezendam NPM, Pijlman B, Bhugwandass C, et al. Chemotherapyinduced peripheral neuropathy and its impact on health-related quality of life among ovarian cancer survivors: results from the population-based PROFILES registry. Gynecol Oncol. 135: 510 517

10. Hershman DL, Lacchetti C, Dworkin RH, et al. (2014) Prevention and management of chemotherapy-induced peripheral neuropathy in survivors of adult cancers: American Society of Clinical Oncology Clinical Practice Guideline. J Clin Oncol: JCO. 2013.2054. 0914

11. Pachman DR, Watson JC, Lustberg MB et al (2014) Management options for established chemotherapy-induced peripheral neuropathy. Support Care Cancer:1-15

12. Müller-Schwefe G, Ahlbeck K, Aldington D et al (2014) Pain in the cancer patient: different pain characteristics CHANGE pharmacological treatment requirements. Curr Med Res Opin 30:1895-1908

13. Bouhassira D, Attal N, Alchaar $\mathrm{H}$ et al (2005) Comparison of pain syndromes associated with nervous or somatic lesions and development of a new neuropathic pain diagnostic questionnaire (DN4). Pain 114:29-36

14. Cleeland C, Ryan K (1994) Pain assessment: global use of the Brief Pain Inventory. Ann Acad Med Singap 23:129-138

15. Young Ho Y, Mendoza TR, Dae Seog H et al (2004) Development of a cancer pain assessment tool in Korea: a validation study of a Korean version of the Brief Pain Inventory. Oncology 66:439-444

16. Park S, Park M, Won J et al (2006) EuroQol and survival prediction in terminal cancer patients: a multicenter prospective study in hospice-palliative care units. Support Care Cancer 14:329-333

17. Kim SH, Kim HJ, Lee S-i, Jo M-W (2012) Comparing the psychometric properties of the EQ-5D-3L and EQ-5D-5L in cancer patients in Korea. Qual Life Res 21:1065-1073

18. Rabin R, Charro Fd EQ-SD: a measure of health status from the EuroQol Group. Ann Med 2001;33: 337-343

19. Vadalouca A, Raptis E, Moka E, Zis P, Sykioti P, Siafaka I (2012) Pharmacological treatment of neuropathic cancer pain: a comprehensive review of the current literature. Pain Pract 12:219-251

20. Mols F, Beijers T, Vreugdenhil G, van de Poll-Franse L (2014) Chemotherapy-induced peripheral neuropathy and its association with quality of life: a systematic review. Support Care Cancer:1-9

21. Cella D, Peterman A, Hudgens S, Webster K, Socinski MA (2003) Measuring the side effects of taxane therapy in oncology. Cancer 98:822-831

22. Driessen CML, de Kleine-Bolt KME, Vingerhoets AJJM, Mols F, Vreugdenhil G (2012) Assessing the impact of chemotherapyinduced peripheral neurotoxicity on the quality of life of cancer patients. Support Care Cancer 20:877-881

23. Griffith K, Couture D, Zhu S et al (2014) Evaluation of chemotherapy-induced peripheral neuropathy using current perception threshold and clinical evaluations. Support Care Cancer 22:1161-1169

24. Kim B-J, Park H-R, Roh H et al (2010) Chemotherapy-related polyneuropathy may deteriorate quality of life in patients with Bcell lymphoma. Qual Life Res 19:1097-1103

25. Ostchega Y, Donohue M, Fox N (1988) High-dose cisplatin-related peripheral neuropathy. Cancer Nurs 11:23-32

26. Sorbe B, Graflund M, Nygren L et al (2012) A phase II study of docetaxel weekly in combination with carboplatin every three weeks as first line chemotherapy in stage IIB-IV epithelial ovarian cancer: neurological toxicity and quality-of-life evaluation. Int $\mathrm{J}$ Oncol 40:773-781 
27. Bennett MI, Rayment C, Hjermstad M, Aass N, Caraceni A, Kaasa S (2012) Prevalence and aetiology of neuropathic pain in cancer patients: a systematic review. Pain 153:359-365

28. Garzon-Rodriguez C, Lyras L, Gayoso LO et al (2013) Cancerrelated neuropathic pain in out-patient oncology clinics: a European survey. BMC Palliat Care 12:41

29. García de Paredes ML, del Moral González F, Martínez del Prado P et al (2011) First evidence of oncologic neuropathic pain prevalence after screening 8615 cancer patients. Results of the On study. Ann Oncol 22:924-930
30. Hershman DL, Lacchetti C, Dworkin RH et al (2014) Prevention and management of chemotherapy-induced peripheral neuropathy in survivors of adult cancers: American Society of Clinical Oncology Clinical Practice Guideline. J Clin Oncol 32:1941-1967

31. Network NCC (2014) NCCN clinical practice guidelines in oncology (NCCN Guidelines $\left.{ }^{\circledR}\right)$ : adult cancer pain. V 2.2014

32. Ripamonti CI, Santini D, Maranzano E, Berti M, Roila F, Group obotEGW (2012) Management of cancer pain: ESMO Clinical Practice Guidelines. Ann Oncol 23:vii139-vii154 